\title{
Lcr1 Immunogen Sequencing and Anti-Leishmaniasis Vaccine Producing
}

\author{
Azimian- moghadam H, Haghshenas M, Mahmoudzaeh-Niknam H3 $\square$ \\ Hanieh Azimian- moghadam, Department of Genetics, Faculty of Science, Science And Research Branch, \\ Islamic Azad University Tehran, Iran (hanie.azimian@yahoo.com) \\ Maryam Haghshenas, Department of Biology, Faculty of Science, University of Guilan, Rasht, Iran \\ (e-mail: m_haghshenass@yahoo.com) \\ Hamid Mahmoudzaeh-Niknam* (Corresponding author), Department of Immunology, Pasteur Institute of Iran, \\ Tehran 13164, Iran (mahmoudh@pasteur.ac.ir)
}

\begin{abstract}
LCRI is an immunogenic molecule discovered from L. chagasi with potential as a component of a Leishmania subunit vaccine. This short review aims to explain Lcrl immunogen sequencing and association of Lcrl with Anti-leishmaniasis vaccine.
\end{abstract}

Keywords: Lcrl, Anti-leshmaniasis vaccine.

\section{Introduction}

LCR1 is an immunogenic molecule discovered from $L$. chagasi with potential as a component of a Leishmania subunit vaccine. If this protein has potentials for being used in a vaccine or diagnostic testing, there should be little variability in this molecule between $L$. infantum isolates from diverse geographic regions [1] .

The use of PCRs has slowly become the preferred way for diagnosing leishmaniasis since conventional parasitological methods are not sufficiently sensitive [2].

It appears to be the most sensitive single diagnostic test for each form of leishmaniasis [3].

Recently, two PCR RFLP assays targeting intergenic regions in nuclear DNA, the SLME and ITS1 PCRs, have been shown to be useful in the diagnosis and identification of pathogenic species of Leishmania.[4],[5]

However, when further parasite characterization is needed, the ITS1 PCR is both highly sensitive and specific and enables one to identify the Leishmania species present in the lesion in a high percentage of cutaneous leishmaniasis cases.[6]

Vaccination with LCR1 in a murine model of Visceral Leishmaniasis has shown some degree of protection against the disease [6]

We were interested in whether LCR1 has potential to be used as a vaccine or a diagnostic molecule in Iranian individuals.

Leishmaniasis is a parasitic disease affecting > 12 million people in 88 countries (66 Old World and 22 New World countries), with 350 million more at risk. The clinical picture of leishmaniasis is heterogeneous and can be classified into three forms: cutaneous (CL), mucocutaneous (MCL), and visceral leishmaniasis (VL).[7]

The clinical pattern of the disease showed variation in the severity and duration and low response to treatment in some cases raising the possibility of the existence of different Leishmania species[8]

At least 21 species and subspecies of Leishmania have been recorded as being infective to humans, many of which cause extensive morbidity and are responsible for a wide spectrum of clinical symptoms. Cutaneous leishmaniasis is a common skin disease in the Middle East region affecting all ages and both sexes. Both CL and VL occur in different parts of Iran. Leishmania tropica, the causative agent of anthroponotic cutaneous leishmaniasis, and L. major, which causes zoonotic cutaneous leishmaniasis (ZCL), are endemic in various regions of Iran with a high incidence rate. CL is still considered an important health problem in many areas of 
the world, especially the Eastern Mediterranean region, and almost all countries of the Middle East, including Iran. In Iran the disease prevalence is high in some foci, including Isfahan,Shiraz,Khorasan, Khuzestan and Kerman provinces.[9]

The history of vaccination with the virulent forms of Leishmania called as leishmanization dates back to early 20th century but due to safety concerns it was banned for trial in human models; first generation vaccine was limited by the immunity conferred by it as it was effective in Sudan (VL) and Iran (CVL) but was not a suitable candidate in Brazil, second generation vaccines are in trial and are useful in providing protection of varying levels in different species along with the DNA and other subunit vaccines.

In present era, majority of vaccine candidates have been tested in mice and hamsters but have provided us with variable results. The major hurdle in developing a potent vaccine is lack of more than one experimental model for studies which do not provide us all the facets of immune responses in humans and the safety issues further limits their development. [10]

L.tropica was cultured and its genomic DNA was extracted. ITS1 PCR and RFLP methods were used to determine the species of the parasite. Lcrl gene was amplified by PCR using specific primers and Taq polymerase. Bands were sequenced after gel electrophoresis. Analysis of the sequences was carried out by chromas software and BLAST.

Our findings show lcrl sequence of $L$. tropica isolate contains important similarities at nucleotide sequence of DNA with L. infant (97\%). High level of conservation increase the possibility for this molecule to be immunogenic in host infected by L. tropica.

The advent of molecular technology in the past 20 years has influenced trends of vaccine development, including that against leishmania. Gene cloning has made it possible to produce larger quantities of pure proteins in vitro, without the need for tedious isolation. Thus vaccine production moved into an era of subunit vaccines instead of the use of whole organisms. In addition, the success of introduction of small DNA sequences coding for specific proteins as a means of correcting effective genes opened up possibilities for the use of DNA vaccines coding for critical immunogenic components of microorganisms as an alternate approach to the direct introduction of microbial proteins.[11]

Access to numerous DNA sequences will favour the development of genetic vaccines over the conventional ones, considering its simple use, low cost of production and flexibility of combining multiple genes in a single construct. [12]

LCR1 is a recombinant protein which shares homology with Trypanosoma cruzi flagellar antigen from $L$. chagasi stimulated proliferation of splenic T lymphocytes from $L$. infantum infected C3H and BALB/c mice and induced IFN-g but not IL-4, IL-5 or IL-10 secretion. Immunization with LCR1 partially protected BALB/c mice against challenge with $L$. infantum[12]

Recombinant antigen (Lcr1) containing homologous 67-amino-acid repeats that stimulated IFN-g but not IL10, IL-4, or IL-5 production by lymphocytes from both susceptible and resistant mice. Lcr1 provided partial protection against challenge with $L$. chagasi, indicating its potential utility in a subunit vaccine.[6]

The nucleotide difference between $L$. infantum and L. tropica may change the antigenicity of the encoded peptide, so immunological studies is needed to determine the level of immunogenicity of this molecule in hosts infected by L. tropica. According to high similarity of lcrl gene in L. infantum to L. tropica, protein product of the $l$ crl gene is potent candidate to vaccine designing against Cutaneous Leishmaniasis.

\section{Acknowledgment}

We appreciate all who helped us to exert this study.

\section{References}

[1] Mahmoudzadeh-Niknam H, Abrishami F, Doroudian M, Moradi M, Alimohammadian MH ,Parvizi P. Complete conservation of an immunogenic gene (lcr1) in Leishmania infantum and Leishmania chagasi isolated from Iran, Spain and Brazil. J Vector Borne Dis.2010;47:204-210.

[2] Bensoussan E, Nasereddin A, Jonas F,Schnur L.F, Jaffe C.L. Comparison of PCR Assays for Diagnosis of Cutaneous Leishmaniasis.Journal of clinical microbiology. 2006; 44(4): 1435-1439.

[3] Pourmohammadi B, Motazedian M.H, Hatam G.R, Kalantari M, Habibi P, Sarkari B. Comparison of Three Methods for Diagnosis of Cutaneous Leishmaniasis. Iranian J Parasitol: 2010; 5(4,): 1-8.

[4] Lachaud L, Marchergui-Hammami S, Chabbert E, Dereure J, Dedet J.P, 
Bastien P. Comparison of six PCR methods using peripheral blood for detection of canine visceral leishmaniasis J. Clin. Microbiol. 2002;40:210-215.

[5] Marfurt J, Nasereddin A, Niederwieser I, Jaffe C.L, Beck H.P, Felger I. Identification and differentiation of Leishmania species in clinical samples by PCR amplification of the miniexon sequence and subsequent restriction fragment length polymorphism analysis. J. Clin. Microbiol. 2003; 41: 3147-3153.

[6] Wilson ME, Young BM, Andersen KP, Weinstock JV, Metwali A, Ali KM, et al. A recombinant Leishmania chagasi antigen that stimulates cellular immune responses in infected mice. Infect. Immun. 1995; 63(5): 2062-2069.

[7] Kumar J, Bumb R, Ansari N, Mehta R, Salotra P.Cutaneous Leishmaniasis caused by leishmania tropica in BIKANER, INDIA: Parasite identification and characterization using molecular and immunologic tools. Raam J.Trop. Med. Hyg. 2007; 76(5): 896-901.

[8] Mahdy M.A.K, Al-Mekhlafi H.M, Al-Mekhlafi A.M, Lim Y.A.L, Bin Shuaib N.O.M, Azazy A.A, et al. Molecular Characterization of Leishmania Species Isolated from Cutaneous Leishmaniasis in Yemen. PLoS ONE.2010; 9(5): 12879 .

[9] Farahmand M, Nahrevanian H, Atashi Shirazi H, Naeimi S, Farzanehnejad Z. An overview of a diagnostic and epidemiologic reappraisal of cutaneous leishmaniasis in Iran. Braz J Infect Dis. 2011; 15(1):17-21.

[10] Singh B, Sundar S. Leishmaniasis: Vaccine candidates and perspectives. Vaccine. 2012; 30: 3834-3842.

[11] Tabbara K.S. Progress toeard leishmania vaccine. Saudi med j.2006; 27(7): 942-950. [12].Khamesipour A, Rafati S, Davoudi N, Maboudi F, Modabber F. Leishmaniasis vaccine candidates for development: A global Overview. Indian J Med Res. 2006; 123: 423-438. 